\title{
A Research on the Planning and Design of RV Camp Based on Self - driving Tourism Experience
}

\author{
Hui Zang ${ }^{a}$, Xiaohui Li ${ }^{b}$ and Dan Huo ${ }^{c}$ \\ Dalian University of Technology, Dalian 116024, China; \\ a106772196@qq.com, blixiaohui12390@126.com, c 19647361@qq.com
}

Keywords: traveling by car; tourism experience; RV camp

\begin{abstract}
With the rapid growth of private car ownership in recent years, the reform of our holiday system and the comprehensive construction of infrastructure such as road traffic provide the necessary conditions for the development of self-driving tourism. Self-Driving this behaviororiented experience of the form of tourism because of its freedom, flexibility, has become the first choice for family-style short-term tourism. However, as the basic service place for traveling by car, the caravan camp is still in the stage of rapid development and the upsurge of building caravan camp in all parts of China. Compared with the mature caravan design and management system in developed countries in Europe and the United States, the design and layout of RV camps in our country is not perfect, the functions are single, the supporting services are relatively backward, and the tourism products are not attractive. Based on the planning and design method of tourism scenic spot and the demand of self - driving travel experience, this paper explores the method of planning and design of RV camp suitable for China's national conditions through the research of planning and design of RV camp in Da ping Village, Long men County, Huizhou, Guangdong.
\end{abstract}

\section{Self-driving travel experience to guide the development of RV camps}

The so-called tourist experience mainly emphasizes the tourists in the process of tourism have a pleasant emotion and then leave a good memory. The car camp is self-driving tourism and camping activities, an important basis for service facilities, is a certain site and facilities conditions to the car as a carrier to supply and rest as the main purpose of environmental facilities. ${ }^{[1]}$ In China because the car is also a small minority of consumer products have not been popular, therefore, China's RV camps are mainly in the form of rental car-based, and more for small family car tourists to provide the service RV camps provide self-drive tourists with a degree of freedom in time and space, and enjoy the pleasure in a relatively unrestricted environment and behavior, in line with the nature of self-drive travel experience.

\section{Comparison to the traditional camping car tourist attractions advantage}

\section{Ecological advantages}

For adapting to the current rapid development of the tourism industry, many natural scenic spots large numbers of traditional resort-style hotel to meet the accommodation needs of tourists. In the process of construction and use of imperfect management, resulting in serious damage to the scenic environment, contrary to the harmonious development of the concept of man and nature. The RV camp has the characteristics of variability, the use of land and landscape resources development intensity is low. Under normal circumstances, the construction of fixed construction is low, mostly exposed and semi-exposed type, even in a considerable area only for the basic road settings, so many require to protect or not allow the construction of the nature of the land area, Become the most ideal way of land use and development. 


\section{Meet the needs of traveling by car travel experience}

Joseph Pine and James Gilmore in the "experience economy" in the experience is divided into four parts - entertainment (Entertainment), education (Education), escape reality (Escape) and aesthetic (Estheticism). ${ }^{[2]}$ Motorhome camp provides a self-driving tourists and entertainment, educational display, return to nature, feel good environment carrier. Self-driving tourists not only in the car camp to get the basic material needs, more importantly, to participate in the camp, experience various types of entertainment, feel the local culture, away from the hustle and bustle of urban environment, return to nature, Into the natural. This is coupled with the nature of the selfdriving tour experience.

\section{The car camp site selection conditions}

As the types of camp sites can be divided into six types: coastal type, island type, lake village type, mountain type, forest type. ${ }^{[3]}$ Regardless of which type of environment, if you want to build a car camp, to meet the following basic conditions.

\section{Social and environmental conditions}

Appropriate to away from the city center, to avoid activities within the camp and the surrounding residents interfere with each other; but the infrastructure should be relatively complete or relatively easy to equipped with drainage, electricity, communications and other related infrastructure conditions; convenient transportation, accessibility.

\section{Natural environmental conditions}

The general choice of scenic, base around a good landscape resources, gentle open terrain, ventilation and drainage is better, the climatic conditions of pleasant land to build the car camp. However, natural landscapes are often accompanied by natural disasters such as landslides, floods and waves in special weather conditions. They should be considered when selecting the location of the camps to avoid personnel and economic losses.

Therefore, within 2 hours around the city, with good natural and cultural resources of the suburban areas and scenic spots for the car camp construction of the ideal plots of choice.

\section{Longmen County Daping Village car camp design interpretation}

Based on the above analysis, a comprehensive evaluation and analysis of the project site of Daping Village car park in Longmen County, Huizhou City, Guangdong Province is conducted.

\section{Social and environmental conditions}

(1) Location analysis of the project

Huizhou City is located on the southeastern part of Guangdong Province, the northeastern part of the Pearl River Delta, the unique natural and geographical conditions. Rich in natural resources, the climate is mild and pleasant, the four seasons for tourism.

(2) External traffic analysis

Longmen County is located in the middle of Guangdong Province, southeast and Heyuan City, Boluo County border, southwest and Conghua City, by the city adjacent to the north and Xinfeng County. The county seat is only 160 kilometers from Guangzhou City, 97 kilometers from Huizhou, and 160 kilometers from Shenzhen, forming a convenient transportation network. Base near the S119 provincial highway. Guanghe Expressway, Longshen Expressway, Longao Expressway, Guanghui Expressway, Huihe Expressway and Huiyuan Expressway. There are planned in the Guangdong-Hunan high-speed, high-speed river, and other freeways in the Longmen crossing to form a dense traffic network. Making the gantry into the Pearl River Delta economic circle within an hour. 
(3) Analysis of tourism resources around the base

Longmen County, a beautiful, charming scenery, very rich in tourism resources, mainly Nankun mountain, Paradise Hill, Longyan, hot springs, and other unique tourist attractions. Especially in hot springs resources, in 2011 became the Ministry of Land and Resources announced the first batch of "hot springs in China town." Longmen County, a unique tourism resources to the local eco-tourism industry to flourish, the tourism industry to determine the dominant position.

\section{Natural environmental conditions}

(1) Climate

The average annual temperature of 22 degrees, evergreen, frost-free period of about 350 days. Climatic conditions are very favorable as camp activities.

(2) Terrain

Base is relatively flat terrain, and a large area of plantation, the vegetation density is not, more suitable for the construction of car camps.

(3) River system

The water area is broad, the river is meandering, the water bank is wide and sometimes narrow, and the water flow speed is slow and acute with the change of the river width. The water area of the Daping village is about 100 meters wide. It is also ideal for water rafting, canoeing and other water recreation projects. Visitors can enjoy the senses while enjoying the beautiful scenery and enrich the tourism experience.

(4) Vegetation

The largest characteristic vegetation in the base is the dense bamboo forest on both sides of the river bank, and the bamboo forest extends to the surface of the water and forms the reflection of the oriental charm. The coastal floodplain grows a variety of aquatic plants to form a natural wetland landscape, And gave birth to a wealth of biological species; close to the land to grow large tracts of cash crops, such as into a piece of orchard for tourists to come to vacation picking activities of the venue; large area of the flower field, creating a unique man-made landscape.

\section{Concept planning}

(1) Planning objectives

Based on the existing conditions, make full use of the advantages of local natural landscape and human resources to protect and highlight the local characteristics of Huizhou, the spirit of "focusing on protection, ecological priority, rational use, healthy development" design principles, to create both eco-tourism, agricultural tourism and human education And other features of the RV campsite. The combination of natural environment and artificial environment, protection and development in harmony with the protection of the original ecological environment and historical and cultural monuments on the basis of the camp through different types of forms, characteristics of ancient villages, country trails, integrated service area, diverse Recreation area and other landscape nodes, forming a point to bring the line with the flow of space for visitors to provide a close to nature, experience the rural life, sports and fitness, daily leisure activities and other places.

(2) Functional partition

Daping Village car camp is divided into camp area, the central service area, the central activity area, ecological agriculture area, historical and cultural exhibition area, ecological protection area six great power area.

(1) Position area

As a result of the diversification of travel modes and the choice of diversified combinations of camp forms, from a simple car camp into a self-driving, self-propelled car, trailer-type car, tent camping, mobile homes and other combinations of camps, In order to maintain the original vegetation, restore and nurture the natural waterfront forest, the camp layout according to different 
environmental selection scattered group settings, to avoid excessive concentration of the use of the land ecology The environment can cause the bad influence.

(2) Central service area

The central service area is equipped with tourist centers, supermarkets, hospitals, restaurants, public health facilities, rental center, shower room, laundry room, car repair and other service facilities to meet the living needs of tourists.

(3) Central activity area

There are various playgrounds, beach-type swimming pools, archery and other amusement facilities in the central activity area. Children's activity areas of different ages can meet the diversified needs of different types of tourists.

(4) Ecological agriculture area

Including ecological pasture, ecological picking garden.

Ecological ranches within the planning set up ecological fish ponds, ecological park, promote ecosystem circulation, increase the human and animal, human and natural interaction, embodies the harmonious coexistence between man and nature.

Ecological picking Park planting green ecological crops, cultivate cash crops, improve local farmers' employment opportunities, enhance the economic value of the original plots, so that tourists can pick in different seasons experience.

(5) Historical and cultural display area

In the preservation of the original appearance of the local village buildings under the premise of the old repair old, fully tap the local food culture, folk arts and crafts, festivals and other intangible cultural heritage, the original town to create a regional cultural characteristics of commercial street, Business forms, enhance the vitality of the ancient street, thereby enhancing the local residents of the economic income and living standards.

\section{(6) Ecological Protection Zone}

There are large tracts of natural bamboo and tropical broad-leaved forest within the base, the planning to take the principle of ecological protection, to maintain its original natural vegetation. Natural forest built within the pedestrian path of the forest, used to guide the orderly forest walkers to prevent random trampling caused by the degradation of vegetation, destruction of the local ecological environment.

\section{Conclusion}

A beautiful environment, complete facilities, activities, rich car camps can bring visitors a pleasant travel experience. Motor car camp is the basis for the further development of self-driving tourism, and self-driving travelers a variety of travel experience needs will guide the future development of China's car camp an important basis. Different areas due to cultural background and lifestyle differences, people's travel experience needs are also different, in-depth study of different regions of China's self-driving travelers diverse experience needs, to build a more scientific, humane and more suited to China's national conditions of the car camp.

\section{References}

[1] LUO Yanning, Car camp planning and design methods --- Nanjing Dashihu eco-tourism resort as an example [J]. Chinese garden, 2007.11.

[2] Joseph .P, James .G. Experience Economy [M]. Beijing: Mechanical Industry Press, 2002.

[3] ZANG Hui, TANG Jian. Research on ecological landscape design of motorhome camp - A case study of Shenyang Qixing Wetland Park caravan [J]. Urban architecture, 2015.11. 